\title{
TAMANHO DA SEMENTE E O TESTE DE ENVELHECIMENTO ACELERADO PARA SOJA
}

\author{
Júlio Marcos Filho' ${ }^{12 *}$; Ana Dionisia Coelho Novembre'; Helena Maria Carmignani Pescarin \\ Chamma $^{1}$ \\ ${ }_{2}^{1}$ Depto. de Produção Vegetal - USP/ESALQ, C.P. 9 - CEP: 13418-900 - Piracicaba, SP. \\ Bolsista CNPq. \\ *Autor correspondente <jmarcos@carpa.ciagri.usp.br>
}

RESUMO: A pesquisa teve como objetivo avaliar a possível relação entre efeitos da utilização de sementes de soja com diferentes tamanhos nos resultados do teste de envelhecimento acelerado. Foram analisados lotes dos cultivares BR-37 e Embrapa 48 (sementes não classificadas e de três tamanhos, classificadas em peneiras de crivos oblongos). Para cada lote e peneira, foram distribuídas três subamostras de $42,5 \mathrm{~g}$ de sementes, em camadas simples ou pré-pesadas $(42,5 \mathrm{~g})$ na superfície da tela metálica de cada compartimento individual (caixa plástica), utilizado como câmara interna para a condução do teste. Cada caixa plástica recebeu $40 \mathrm{~mL}$ de água (constituindo ambiente com 100\% U.R. do ar) ou $40 \mathrm{~mL}$ de solução saturada de cloreto de sódio (ambiente com $76 \%$ U.R.). Estudaram-se os períodos de 48 e 72 horas de envelhecimento das sementes, em $\mathrm{BOD}, \mathrm{a} 41^{\circ} \mathrm{C}$. O teste de germinação subseqüente foi avaliado no quarto dia após a semeadura. A distribuição de amostras com massa uniforme não elimina os efeitos do tamanho das sementes. Desta maneira, o teste de envelhecimento acelerado, em sementes de soja, fornece informações mais consistentes quando as amostras comparadas são constituídas por sementes de tamanho semelhante. O uso de solução salina torna o teste menos severo, mas não reduz sua eficiência.

Palavras-chave: Glycine max, semente, análise, potencial fisiológico, vigor

\section{SEED SIZE AND THE ACCELERATED AGING VIGOR TEST FOR SOYBEAN}

\begin{abstract}
The accelerated aging test is one of the most useful soybean seed vigor tests. This test exposes seeds to a stress of high relative humidity $(100 \%)$ and high temperature $\left(41^{\circ} \mathrm{C}\right)$ for 48 or $72 \mathrm{~h}$. The main purpose of this study was to determine whether seed size affects water uptake during the accelerated aging test and its influence on the results of this test. Soybean seed lots from cultivars BR-37 and Embrapa 48 were separated

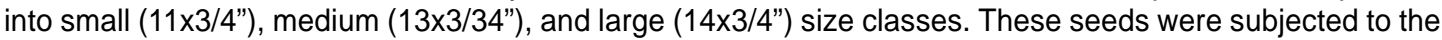
germination and accelerated aging tests; this test was conducted as recommended by the Association of Official Seed Analysts, and also the "saturated salt accelerated aging" procedure. Small seeds had higher water content compared to larger seeds, after accelerated aging, even when an uniform weight of seeds was placed on the inner tray during accelerated aging. Seed analysts must consider differences in soybean seed size when conducting and interpreting results from the accelerated aging vigor test. The salt saturated accelerated aging procedure has potential application for soybean seed vigor evaluation.

Key words: Glycine max, seed analysis, physiological quality, vigor
\end{abstract}

\section{INTRODUÇÃO}

O teste de envelhecimento acelerado, um dos mais indicados para a avaliação do vigor de sementes, foi desenvolvido por Delouche (1965), visando estimar o potencial de armazenamento de sementes de trevo e de festuca, partindo da hipótese de que a morte das sementes, durante o armazenamento, seria causada pela coagulação de proteínas e que a elevação da temperatura, acima de certos limites, acelerava o processo de deterioração de sementes.

O princípio desse envelhecimento estabelece que a taxa de deterioração é acelerada consideravelmente, quando as sementes são expostas a temperatura e umidade relativa elevadas; estas são consideradas como fatores ambientais preponderantes na intensidade e velocidade de deterioração. Assim, verifica-se que as amostras com baixo vigor apresentam queda mais acentuada da viabilidade, quando submetidas às condições do teste, enquanto as mais vigorosas geralmente são menos afetadas em sua capacidade de produzir plântulas normais.

Embora o teste de envelhecimento acelerado tenha sido considerado padronizado para avaliar o vigor de sementes de soja (TeKrony, 1995), ainda há aspectos específicos da metodologia que merecem elucidação. Por exemplo, sabe-se que os lotes de sementes de soja normalmente são constituídos por materiais de diferentes tamanhos. Essa variação, observada dentro de cada lote e entre cultivares, tem causado dúvidas quanto ao grau de influência desse parâmetro sobre os resultados do teste de envelhecimento acelerado. Essa preocupação é justificada porque as sementes menores, com maior área de exposição, 
geralmente captam água mais rapidamente e, assim, podem ser mais prejudicadas pelas condições impostas no teste e provocar distorções nas informações obtidas.

Uma alternativa para a solução desse problema foi proposta por Tomes et al. (1988), recomendando o uso de amostras com massa pré-determinada e uniforme (40 a $45 \mathrm{~g}$ ), em vez de distribuí-las formando camada simples na superfície da tela metálica colocada no interior do compartimento individual (caixa plástica) utilizado como câmara interna para a condução do teste.

Essa proposta tem sido aceita em laboratórios dos EUA e, também, no Brasil, embora a maioria dos pesquisadores não leve em consideração a definição do que seriam as sementes "grandes" ou "pequenas" e essa variação seja comum entre lotes do mesmo ou de diferentes cultivares. De acordo com Wetzel (1978), as sementes de soja com maior potencial fisiológico estariam compreendidas dentro dos limites $M \pm 2 / 64$ " $(M \pm 0,8 \mathrm{~mm})$, sendo $\mathrm{M}$ a largura ou a espessura média das sementes que compõem o lote. Esse fato é extremamente importante, permitindo direcionar a classificação pelo tamanho, durante o beneficiamento, prática utilizada pela maioria das empresas produtoras de sementes de soja, no Brasil; essa iniciativa favorece significativamente a precisão da semeadura (Krzyzanowski et al., 1991).

Acrescente-se que a prática tem demonstrado que os objetivos da recomendação efetuada por Tomes et al. (1988) podem não ser completamente atingidos; essa possibilidade foi confirmada por Fahim \& McDonald ${ }^{1}$ que, trabalhando com sementes de soja, verificaram diferenças entre o comportamento de sementes "grandes" e "pequenas" no teste de envelhecimento acelerado.

Por outro lado, a exposição das sementes à umidade relativa de, aproximadamente, $100 \%$ durante 0 teste, aliada à temperatura elevada $\left(41^{\circ} \mathrm{C}\right)$, contribui para a proliferação de microrganismos (principalmente fungos de armazenamento), principalmente em amostras de potencial fisiológico inferior; essa situação pode causar variação adicional dos resultados, provocada por fatores não inerentes às sementes. Para atenuar o problema, Jianhua \& McDonald (1996), trabalhando com sementes de floríferas, e Panobianco \& Marcos Filho (1998), com pimentão, verificaram que o uso de soluções salinas, em substituição à água no interior do compartimento individual (câmara interna), permitiu a condução do teste em ambiente com 55 a $87 \%$ de umidade relativa, acarretando a absorção mais lenta de vapor d'água e deterioração mais lenta sem afetar a consistência dos resultados obtidos.

Diante do exposto, o presente trabalho teve como objetivos estabelecer relações entre a caracterização do tamanho das sementes e os resultados do teste de envelhecimento acelerado e verificar a viabilidade da utilização de solução salina e da conseqüente redução da umidade relativa do ar, no interior de cada compartimento individual utilizado para a condução do teste.

\section{MATERIAL E MÉTODOS}

A pesquisa foi conduzida, em dois anos experimentais (1998/99 e 1999), com dois cultivares de soja (BR-37 e Embrapa 48), cada um representado por diferentes lotes de sementes, não classificadas pelo tamanho e com potenciais fisiológicos distintos. Nos dois anos, foram utilizadas sementes recémcolhidas.

Após a recepção, foi efetuada a análise da distribuição do tamanho das sementes de cada lote. Para tanto, duas amostras de $200 \mathrm{~g}$ de cada lote e cultivar foram separadas em uma seqüência de peneiras de crivos oblongos, superpostas em ordem decrescente, de $17 \times 3 / 4 "(6,74 \times 19,05 \mathrm{~mm})$ a $10 \times 3 / 4 "(3,96 \times 19,05 \mathrm{~mm})$, respeitando-se a diferença de 1/64" $(0,4 \mathrm{~mm})$ entre a largura dos crivos. Completadas essas separações, efetuou-se a pesagem das sementes retidas em cada peneira (precisão de $0,1 \mathrm{~g}$ ) e a determinação das percentagens de retenção.

Essa análise forneceu subsídios para a escolha dos tamanhos das sementes utilizadas no presente estudo. Assim, após as determinações das percentagens de retenção, em cada peneira e, conhecendo-se o tamanho médio (M) das sementes de cada lote (Wetzel, 1978), o trabalho foi conduzido com materiais de três tamanhos, ou sejam: no primeiro ano experimental, para o cultivar BR-37, as sementes "grandes" (G), "médias" (M) e "pequenas" $(P)$ corresponderam, respectivamente, às retidas na peneira $13 \times 3 / 4$ " $(5,16 \times 19,05 \mathrm{~mm}), 12 \times 3 / 4$ " $(4,76 \times 19,05 \mathrm{~mm})$ e $10 \times 3 / 4(3,96 \times 10,05 \mathrm{~mm})$. No segundo ano e para o cultivar Embrapa 48, nos dois anos, foram representadas pelas sementes retidas nas peneiras

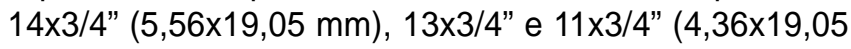
$\mathrm{mm})$, respectivamente. O quarto tratamento foi representado por sementes dos materiais originais de cada lote e cultivar, não classificados pelo tamanho, nos dois anos experimentais.

Além da determinação da massa de 1000 sementes de cada tratamento, de acordo com as Regras para Análise de Sementes (Brasil, 1992), foram realizados os seguintes testes, nos dois anos experimentais:

\section{Germinação}

Conduzido com quatro repetições para cada tratamento, em rolos de papel-toalha, a $25^{\circ} \mathrm{C}$; cada rolo com 50 sementes foi considerado como uma subamostra. Visando ao umedecimento uniforme, a quantidade de água adicionada ao papel correspondeu a 2,5 vezes o peso do substrato. Os testes foram interpretados aos quatro e sete dias após a semeadura, computando-se a percentagem de plântulas normais para cada tratamento, por lote e cultivar. A análise estatística considerou os dados obtidos na contagem efetuada aos sete dias. 


\section{Envelhecimento acelerado}

Estudo da distribuição das sementes sobre a tela metálica da caixa plástica - foram utilizadas caixas plásticas $(11 \times 11 \times 3 \mathrm{~cm})$ como compartimento individual, contendo $40 \mathrm{~mL}$ de água (Marcos Filho et al., 1987). As amostras foram distribuídas de duas maneiras, na superfície da tela metálica: na primeira, as sementes de cada tratamento (P, M, G e material original) constituíram uma camada única, tomando toda a superfície da tela, independentemente do número e do peso das sementes; na segunda, amostras de $42,5 \mathrm{~g}$, para cada tratamento, ocuparam a superfície da tela.

Estudo da substituição da água por solução salina - a umidade relativa no interior do compartimento individual foi determinada pela adição de $40 \mathrm{~mL}$ de água, para obtenção de, aproximadamente, 100\% U.R. (sistema tradicional) ou de $40 \mathrm{~mL}$ de solução saturada de cloreto de sódio ( $76 \%$ U.R.) ao fundo de cada caixa plástica, conforme Jianhua \& McDonald (1996).

Para esses dois estudos, os períodos de permanência das amostras no interior da câmara, a $41^{\circ} \mathrm{C}$, foram de 48 e de 72 horas, seguindo-se a condução de teste de germinação, conforme descrição anterior, interpretado no quarto dia após a semeadura.

\section{Grau de umidade}

Avaliado antes e após cada período de envelhecimento acelerado, para verificação da uniformidade das condições utilizadas para ambos os testes, de acordo com as especificações das Regras para Análise de Sementes (Brasil, 1992).

\section{Procedimento estatístico}

As análises dos dados referentes aos testes de germinação e envelhecimento acelerado foram efetuadas separadamente para cada cultivar, estudando-se os efeitos de lotes e tamanho das sementes, com os tratamentos distribuídos inteiramente ao acaso, segundo esquema fatorial. Nos estudos relativos ao teste de envelhecimento acelerado, esquemas independentes incluíram os efeitos do tipo de distribuição das amostras sobre a tela metálica ou da umidade relativa (água e solução salina) no interior do compartimento individual. Os dados foram transformados para arc sen $\sqrt{\%} \%$ e, as médias, comparadas pelo método de Tukey $(p<0,05)$.

\section{RESULTADOS E DISCUSSÃO}

\section{Análise da distribuição do tamanho das sementes}

A distribuição do tamanho das sementes de cada

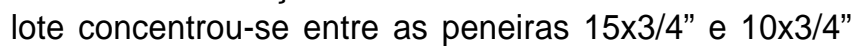
para ambos os cultivares. Isto permite supor que a escolha das peneiras utilizadas para a limpeza dos lotes, com o uso da máquina de ventiladores e peneiras, foi efetuada de modo a obter uma pré-classificação dos lotes quanto à espessura das sementes. Essa operação foi realizada na unidade de beneficiamento das empresas que produziram as sementes dos dois cultivares utilizados nesta pesquisa.

A hipótese dessa provável pré-classificação ou, pelo menos, da tentativa da obtenção de lotes compostos por sementes de tamanho relativamente uniforme, é reforçada pelos resultados das determinações das percentagens de retenção de sementes em cada peneira, em ambos os cultivares. Assim, constatou-se visível concentração de sementes com tamanho próximo ao médio de cada lote (TABELAS 1 e 2). Por essa razão, não foi possível a utilização de sementes cujos tamanhos se encontravam dentro dos limites $M \pm 3 / 64$ ", pois não seria obtida quantidade suficiente de sementes para a execução dos testes programados. Conforme destacou Wetzel (1978), as sementes de soja com potencial fisiológico mais elevado estão compreendidas entre os limites $M+2 / 64$ " e $\mathrm{M}-2 / 64$ ", sendo M o tamanho médio das sementes que compõem o lote. De qualquer maneira, a pesquisa foi conduzida com a utilização de lotes em que a diferença entre as espessuras extremas das sementes era de $3 / 64$ "

TABELA 1 - Cultivar BR-37: distribuição percentual da retenção de sementes de nove lotes em peneiras de crivos oblongos. Piracicaba, 1998/99.

\begin{tabular}{|c|c|c|c|c|c|c|c|c|}
\hline \multirow{2}{*}{ Lotes } & \multicolumn{8}{|c|}{ Peneiras } \\
\hline & $16 \times 3 / 4 "$ & $15 \times 3 / 4 "$ & $14 \times 3 / 4 "$ & $13 \times 3 / 4 "$ & $12 \times 3 / 4 "$ & $11 \times 3 / 4 "$ & $10 \times 3 / 4 "$ & Fundo \\
\hline \multicolumn{9}{|c|}{ 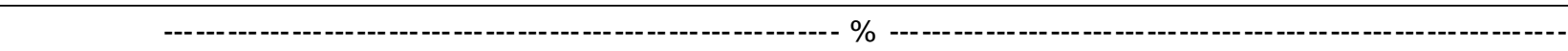 } \\
\hline 1 & --------- & 0,2 & 4,2 & 36,8 & 37,5 & 12,8 & 1,5 & 7,0 \\
\hline 2 & -------- & 0,1 & 1,4 & 26,5 & 45,7 & 20,2 & 1,4 & 4,7 \\
\hline 3 & --------- & 0,1 & 0,7 & 16,9 & 36,6 & 35,0 & 5,7 & 4,9 \\
\hline 4 & --------- & 1,8 & 16,6 & 48,9 & 19,8 & 5,3 & 0,6 & 7,0 \\
\hline 5 & --------- & 0,5 & 8,8 & 44,8 & 31,7 & 10,7 & 1,4 & 2,1 \\
\hline 6 & --------- & 0,9 & 7,4 & 42,7 & 32,0 & 12,7 & 1,9 & 2,4 \\
\hline 7 & --------- & 0,9 & 6,9 & 43,2 & 31,1 & 13,0 & 2,1 & 2,7 \\
\hline 8 & 0,1 & 0,3 & 7,5 & 44,5 & 30,8 & 12,1 & 2,5 & 2,2 \\
\hline 9 & 0,2 & 1,5 & 14,9 & 43,2 & 28,3 & 10,9 & 0,3 & 0,7 \\
\hline
\end{tabular}

Scientia Agricola, v.57, n.3, p.473-482, jul./set. 2000 
TABELA 2 - Cultivar EMBRAPA 48: distribuição percentual da retenção de sementes de sete lotes em peneiras de crivos oblongos. Piracicaba, 1998/99.

\begin{tabular}{|c|c|c|c|c|c|c|c|c|}
\hline \multirow{2}{*}{ Lotes } & \multicolumn{8}{|c|}{ Peneiras } \\
\hline & $16 \times 3 / 4 "$ & $15 \times 3 / 4 "$ & $14 \times 3 / 4 "$ & $13 \times 3 / 4 "$ & $12 \times 3 / 4 "$ & $11 \times 3 / 4 "$ & $10 \times 3 / 4 "$ & Fundo \\
\hline & $-\cdots---$ & $\cdots$ & - & - & --.-- n n & 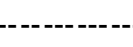 & --- & ------ \\
\hline 1 & 0,1 & 2,8 & 12,8 & 39,3 & 29,6 & 12,6 & 0,9 & 1,9 \\
\hline 2 & 0,5 & 5,5 & 20,5 & 41,0 & 22,2 & 8,0 & 1,0 & 1,3 \\
\hline 3 & 0,1 & 0,6 & 6,6 & 38,3 & 29,2 & 14,3 & 2,1 & 8,8 \\
\hline 4 & 0,1 & 0,9 & 12,4 & 50,4 & 26,0 & 8,2 & 1,0 & 1,0 \\
\hline 5 & 0,2 & 1,2 & 14,4 & 49,9 & 24,4 & 8,4 & 0,8 & 0,7 \\
\hline 6 & 0,4 & 1,0 & 13,8 & 47,6 & 27,3 & 7,4 & 1,5 & 1,0 \\
\hline 7 & 0,1 & 0,9 & 12,1 & 48,5 & 28,1 & 8,3 & 1,1 & 0,9 \\
\hline
\end{tabular}

(13 e 10, para 'BR-37'; 14 e 11, para 'Embrapa 48'), suficiente para permitir a detecção de efeitos do tamanho das sementes sobre a resposta aos testes para a avaliação do potencial fisiológico. Sabe-se que várias empresas produtoras de sementes de soja promovem o descarte das retidas na peneira $10 \times 3 / 4 "$, durante o beneficiamento, não considerando os limites verificados por Wetzel (1978).

A separação das sementes por diferenças de tamanho também correspondeu a uma separação pela massa, que se mostrou proporcional às respectivas classes de tamanho (dados não apresentados). As sementes de 'Embrapa 48' possuíam massa mais elevada que as correspondentes de 'BR-37', inclusive quando se consideraram os materiais não classificados, identificados como "original". Essa ocorrência pode ter sido devida à predominância de condições climáticas mais favoráveis nos campos de produção de 'Embrapa 48', em especial durante o período de transferência de matéria seca da planta para as sementes (Egli, 1990; Vieira et al., 1992). No entanto, deve ser também considerado que a descrição das características do cultivar BR-37 indica sementes com massa média de 140 $\mathrm{g} / 1000$ sementes, enquanto as de 'Embrapa 48' apresentam $154 \mathrm{~g} / 1000$ sementes (Embrapa, 1996); cabe ressaltar, porém, que no segundo ano experimental as sementes dos dois cultivares apresentaram tamanhos e pesos semelhantes, dentro de cada fração considerada.

\section{Testes conduzidos com sementes de 'BR-37'}

Germinação - os lotes 5, 6, 7 e 8 apresentaram médias significativamente superiores aos lotes 3 e 4, enquanto o lote 1 geralmente ocupou posição intermediária. Os lotes 2 e 9 exibiram comportamento próximo ao dos de melhor qualidade (TABELA 3). Deve ser destacado que seis dos nove lotes avaliados possuíam poder germinativo superior ao mínimo estabelecido para comercialização de sementes de soja. Por outro lado, as sementes retidas na peneira 10×3/4" apresentaram desempenho inferior, em sete dos nove lotes analisados.

Scientia Agricola, v.57, n.3, p.473-482, jul./set. 2000
TABELA 3 - Cultivar BR-37. Germinação: dados obtidos para os efeitos de lotes, de tamanhos das sementes e coeficiente de variação. Piracicaba, 1998/99.

\begin{tabular}{|c|c|c|c|c|}
\hline \multirow[t]{2}{*}{ Lotes } & \multicolumn{4}{|c|}{ Tamanhos } \\
\hline & Original & 13 & 12 & 10 \\
\hline \multicolumn{5}{|c|}{---- } \\
\hline 1 & $79 \mathrm{BCab}\left(^{*}\right)$ & 83 BCab & $85 \mathrm{ABa}$ & $70 \mathrm{BCDb}$ \\
\hline 2 & $94 \mathrm{Aa}$ & $87 \mathrm{ABCa}$ & $91 \mathrm{Aa}$ & $87 \mathrm{Aa}$ \\
\hline 3 & $71 \mathrm{Ca}$ & $75 \mathrm{Ca}$ & $71 \mathrm{Ba}$ & $79 \mathrm{ABCa}$ \\
\hline 4 & $77 \mathrm{BCa}$ & $81 \mathrm{Ca}$ & $75 \mathrm{Ba}$ & $56 \mathrm{Db}$ \\
\hline 5 & $93 \mathrm{Aab}$ & $97 \mathrm{Aa}$ & $93 \mathrm{Aab}$ & $84 \mathrm{ABb}$ \\
\hline 6 & $94 \mathrm{Aab}$ & $95 \mathrm{ABa}$ & $91 \mathrm{Aab}$ & $85 \mathrm{ABb}$ \\
\hline 7 & $94 \mathrm{Aab}$ & $96 \mathrm{Aa}$ & $93 \mathrm{Aab}$ & $87 \mathrm{Ab}$ \\
\hline 8 & $94 \mathrm{Aa}$ & $95 \mathrm{ABa}$ & $92 \mathrm{Aa}$ & $84 \mathrm{ABb}$ \\
\hline 9 & $94 \mathrm{Aa}$ & $95 \mathrm{ABa}$ & $92 \mathrm{Aa}$ & $84 \mathrm{ABb}$ \\
\hline C.V. (\%) & \multicolumn{4}{|c|}{6,4} \\
\hline
\end{tabular}

$\left(^{*}\right)$ Letras maiúsculas: comparações dentro de cada coluna; letras minúsculas: comparações dentro de cada linha (Teste de Tukey, $5 \%)$.

Envelhecimento acelerado (48 horas) - como esperado, esse teste foi mais sensível que o de germinação para detectar diferenças no potencial fisiológico das sementes. Como exemplo, o lote 2, identificado como de boa qualidade, no teste de germinação, não mostrou o mesmo desempenho no teste de envelhecimento acelerado; os lotes 1 e 9 também ocuparam posição intermediária. A distribuição das amostras de acordo com a massa $(42,5 \mathrm{~g})$ não alterou acentuadamente o posicionamento dos lotes quanto ao vigor. No entanto, as diferenças entre as médias se estreitaram.

Os efeitos do tamanho das sementes foram pronunciados. A comparação entre as médias obtidas para cada lote (TABELAS 4 e 5) permite verificar que, em ambos os procedimentos para a distribuição de amostras, as sementes retidas na peneira $10 \times 3 / 4$ " foram menos vigorosas, em sete dos nove lotes, acompanhando os resultados de germinação. 
TABELA 4 - Cultivar BR-37. Envelhecimento acelerado: dados obtidos para os efeitos de lotes e tamanho das sementes, em testes conduzidos com sementes pré-pesadas, durante 48 horas a $41^{\circ} \mathrm{C}$ e diferentes condições de umidade relativa do ar. Piracicaba, 1998/99.

\begin{tabular}{|c|c|c|c|c|c|c|c|c|}
\hline \multirow[t]{2}{*}{ Lotes } & \multicolumn{4}{|c|}{ Sistema Tradicional } & \multicolumn{4}{|c|}{ Solução Salina } \\
\hline & Orig. & 13 & 12 & 10 & Orig. & 13 & 12 & 10 \\
\hline & \multicolumn{8}{|c|}{ - } \\
\hline 1 & $70 \mathrm{Bca}$ & $71 \mathrm{CDa}$ & 77BCDa & $68 \mathrm{ABa}$ & $76 \mathrm{Bb}$ & $72 \mathrm{Cb}$ & $88 \mathrm{Aa}$ & $67 \mathrm{BCb}$ \\
\hline 2 & $75 \mathrm{Ba}$ & $75 \mathrm{BCa}$ & $68 \mathrm{Da}$ & $59 \mathrm{ABb}$ & $88 \mathrm{Aa}$ & $67 \mathrm{BCb}$ & $87 \mathrm{Aa}$ & $80 \mathrm{Aba}$ \\
\hline 3 & $49 \mathrm{CDa}$ & $53 \mathrm{Da}$ & $47 \mathrm{Ea}$ & $49 \mathrm{BCa}$ & $61 \mathrm{Da}$ & $57 \mathrm{Da}$ & $55 \mathrm{Da}$ & $59 \mathrm{CDa}$ \\
\hline 4 & $50 \mathrm{Da}$ & $50 \mathrm{Da}$ & $45 \mathrm{Ea}$ & $36 \mathrm{Cb}$ & $67 \mathrm{CDa}$ & $77 \mathrm{BCa}$ & $67 \mathrm{Ca}$ & $49 \mathrm{Db}$ \\
\hline 5 & $92 \mathrm{Aa}$ & $94 \mathrm{Aa}$ & $93 \mathrm{Aa}$ & $76 \mathrm{Ab}$ & $93 \mathrm{Aa}$ & $95 \mathrm{Aa}$ & $92 \mathrm{Aa}$ & $82 \mathrm{Ab}$ \\
\hline 6 & $87 \mathrm{ABa}$ & $89 \mathrm{ABa}$ & $89 \mathrm{ABa}$ & $76 \mathrm{Ab}$ & $93 \mathrm{Aa}$ & $92 \mathrm{Aa}$ & $92 \mathrm{Aa}$ & $76 \mathrm{Ab}$ \\
\hline 7 & $92 \mathrm{Aa}$ & $87 \mathrm{ABCab}$ & $88 \mathrm{ABab}$ & $77 \mathrm{Ab}$ & $90 \mathrm{Aa}$ & $89 \mathrm{Aba}$ & $91 \mathrm{Aa}$ & $77 \mathrm{ABb}$ \\
\hline 8 & $88 \mathrm{ABa}$ & $89 \mathrm{ABa}$ & $92 \mathrm{ABa}$ & $77 \mathrm{Ab}$ & $90 \mathrm{Aa}$ & $93 \mathrm{Aa}$ & $87 \mathrm{Aab}$ & $83 A b$ \\
\hline 9 & $74 \mathrm{Bab}$ & $77 \mathrm{BCa}$ & $69 \mathrm{CDab}$ & $60 \mathrm{ABb}$ & $79 \mathrm{Bab}$ & $86 \mathrm{ABa}$ & $71 \mathrm{BCb}$ & $53 \mathrm{CDC}$ \\
\hline
\end{tabular}

$\left(^{\star}\right)$ Letras maiúsculas: comparações dentro de cada coluna; letras minúsculas: comparações dentro de cada linha, para cada condição de umidade relativa do ar (Teste de Tukey, $5 \%$ ).

TABELA 5 - Cultivar BR-37. Envelhecimento acelerado: dados obtidos para os efeitos de lotes e tamanho das sementes, em testes conduzidos com amostras distribuídas em camada única, durante 48 horas a $41^{\circ} \mathrm{C}$ e diferentes condições de umidade relativa do ar. Piracicaba, 1998/99.

\begin{tabular}{|c|c|c|c|c|c|c|c|c|}
\hline \multirow[t]{2}{*}{ Lotes } & \multicolumn{4}{|c|}{ Sistema Tradicional } & \multicolumn{4}{|c|}{ Solução Salina } \\
\hline & Orig. & 13 & 12 & 10 & Orig. & 13 & 12 & 10 \\
\hline 1 & $77 \mathrm{R} C \mathrm{P}$ & $75 \mathrm{Ro}$ & $77 \mathrm{RC} \Omega$ & $67 \mathrm{ABa}$ & $84 \mathrm{AR}$ ? & $85 \mathrm{BCD}$ & $81 \mathrm{cDF}$ & $76 \mathrm{ABh}$ \\
\hline 2 & $72 \mathrm{CDEa}$ & $78 \mathrm{Ba}$ & $79 \mathrm{ABCa}$ & $73 \mathrm{ABa}$ & $74 \mathrm{Bb}$ & $90 \mathrm{BCDa}$ & $87 \mathrm{ABCDa}$ & $83 \mathrm{Aab}$ \\
\hline 3 & $62 \mathrm{DEa}$ & $55 \mathrm{Cab}$ & 51 DEab & $41 \mathrm{Cb}$ & $74 \mathrm{Bab}$ & $83 \mathrm{CDa}$ & $84 \mathrm{BCDEa}$ & $65 \mathrm{BCb}$ \\
\hline 4 & $57 \mathrm{Ea}$ & $51 \mathrm{Cab}$ & $43 \mathrm{~Eb}$ & $29 \mathrm{Cc}$ & $76 \mathrm{Ba}$ & $79 \mathrm{Da}$ & $73 \mathrm{Ea}$ & $58 \mathrm{Cb}$ \\
\hline 5 & $93 \mathrm{Aa}$ & $92 \mathrm{Aa}$ & $83 \mathrm{ABb}$ & $75 \mathrm{ABb}$ & $91 \mathrm{Aa}$ & $93 \mathrm{BCa}$ & $90 \mathrm{ABCa}$ & $79 \mathrm{ABb}$ \\
\hline 6 & $84 \mathrm{ABCab}$ & $93 \mathrm{Aa}$ & $78 \mathrm{ABCb}$ & $75 \mathrm{ABb}$ & $92 \mathrm{Abc}$ & $99 \mathrm{Aa}$ & $94 \mathrm{Aa}$ & $83 \mathrm{Ac}$ \\
\hline 7 & $87 \mathrm{ABab}$ & $92 \mathrm{Aa}$ & $91 \mathrm{Aa}$ & $79 \mathrm{Ab}$ & $89 \mathrm{Aa}$ & $92 \mathrm{BCa}$ & $90 \mathrm{ABCa}$ & $77 \mathrm{ABb}$ \\
\hline 8 & $86 \mathrm{ABa}$ & $91 \mathrm{Aa}$ & $88 \mathrm{ABa}$ & $74 \mathrm{ABb}$ & $89 \mathrm{Aab}$ & $94 \mathrm{ABa}$ & $93 \mathrm{ABa}$ & $83 \mathrm{Ab}$ \\
\hline 9 & 66 DEab & $76 \mathrm{Ba}$ & $65 \mathrm{CDab}$ & $60 \mathrm{Bc}$ & $83 \mathrm{ABab}$ & $89 \mathrm{BCDa}$ & 79 DEab & $76 \mathrm{ABb}$ \\
\hline
\end{tabular}

$\left(^{\star}\right)$ Letras maiúsculas: comparações dentro de cada coluna; letras minúsculas: comparações dentro de cada linha, para cada condição de umidade relativa do ar (Teste de Tukey, $5 \%$ ).

O grau de umidade das sementes maiores (13×3/4") superou em $0,8 \%$ o das menores, após o envelheci-mento conduzido com o uso de amostras distribuídas após pesagem; ao final do período de envelhecimento sob umidade relativa próxima a $100 \%$, os graus de umidade das sementes das peneiras 10 e $13 \times 3 / 4$ " se situaram entre 25,2 e $26,0 \%$, respectivamente; o mesmo ocorreu quando as amostras formaram camada simples (graus de umidade entre $25,9 \%$ e $26,3 \%$ ). Isto pode não ter sido suficiente para justificar o pior desempenho das sementes menores, mas contraria as observações efetuadas por Tomes et al. (1988), segundo as quais o uso de amostras com peso uniforme seria necessário para anular os efeitos do tamanho das sementes sobre o seu comportamento no teste de envelhecimento acelerado.
O uso de solução salina e a conseqüente redução da umidade relativa do ar no interior dos compartimentos individuais contribuiu, de maneira acentuada, para que os efeitos do envelhecimento acelerado se tornassem menos drásticos. As sementes captaram quantidades significativamente menores de água durante as 48 horas de exposição à temperatura elevada $\left(41^{\circ} \mathrm{C}\right)$, atingindo graus de umidade de $12,2 \%$ a $12,4 \%$ ao final desse período, independentemente do sistema de distribuição de amostras.

Apesar desse fato, o teste de envelhecimento acelerado conduzido sob umidade relativa inferior não se tornou menos eficiente na detecção de diferenças entre os potenciais fisiológicos dos lotes avaliados. Assim, as informações quanto ao desempenho dos lotes de melhor 
qualidade $(5,6,7$ e 8$)$, dos intermediários (1, 2 e 9) e dos menos vigorosos ( 3 e 4 ), foram condizentes com as obtidas através do procedimento tradicional (TABELAS 4 e 5). O mesmo ocorreu para a análise dos efeitos do tamanho das sementes; as médias referentes à peneira $10 \times 3 / 4$ " foram significativamente inferiores, em sete dos nove lotes.

Dessa maneira, constatou-se, de um modo geral, que a distribuição das sementes com massa uniforme não evitou a manifestação dos efeitos do tamanho das sementes sobre a sua resposta às condições de ambiente, durante o teste de envelhecimento acelerado. Essa observação se aplica tanto à detecção de diferenças entre lotes como ao desempenho das sementes menores.
Envelhecimento acelerado (72 horas) - a utilização do período de 72 horas também se mostrou eficiente para a identificação de lotes com potencial fisiológico mais elevado, de qualidade intermediária e menos vigorosos. Com pequenas variações, esses lotes foram os mesmos mencionados na exposição dos resultados referentes ao período de 48 horas. No entanto, o período de 72 horas provocou reduções mais drásticas da germinação, principalmente para os lotes 2, 3 e 4 (TABELAS 6 e 7).

Essas manifestações se evidenciaram quando as sementes foram expostas à umidade relativa próxima à saturação do ar, mas também foram notadas com o uso de solução salina. Neste caso, porém, detectaram-se

TABELA 6 - Cultivar BR-37. Envelhecimento acelerado: dados obtidos para os efeitos de lotes e tamanho das sementes, em testes conduzidos com amostras pré-pesadas, durante 72 horas a $41^{\circ} \mathrm{C}$ e diferentes condições de umidade relativa do ar. Piracicaba, 1998/99.

\begin{tabular}{|c|c|c|c|c|c|c|c|c|}
\hline \multirow[t]{2}{*}{ Lotes } & \multicolumn{4}{|c|}{ Sistema Tradicional } & \multicolumn{4}{|c|}{ Solução Salina } \\
\hline & Orig. & 13 & 12 & 10 & Orig. & 13 & 12 & 10 \\
\hline & \multicolumn{8}{|c|}{ - } \\
\hline 1 & $45 \mathrm{CDa}$ & $54 \mathrm{Ca}$ & $43 \mathrm{Ca}$ & $47 \mathrm{Ca}$ & $75 \mathrm{Ba}$ & $74 \mathrm{BCa}$ & $79 \mathrm{ABa}$ & $71 \mathrm{Ba}$ \\
\hline 2 & $25 \mathrm{Eab}$ & $29 \mathrm{Da}$ & $17 \mathrm{Db}$ & $15 \mathrm{Ec}$ & $80 \mathrm{ABa}$ & $78 \mathrm{Ba}$ & $75 \mathrm{Bab}$ & $67 \mathrm{Bb}$ \\
\hline 3 & $09 \mathrm{Fa}$ & $14 \mathrm{Ea}$ & $09 \mathrm{Ea}$ & $11 \mathrm{Ea}$ & $64 \mathrm{Ba}$ & $68 \mathrm{Ca}$ & $65 \mathrm{Ca}$ & $55 \mathrm{Cb}$ \\
\hline 4 & $20 \mathrm{Ea}$ & $19 \mathrm{Ea}$ & 15 DEab & $09 \mathrm{~Eb}$ & $75 \mathrm{Ba}$ & $71 \mathrm{Bc}$ & $72 \mathrm{BCa}$ & $51 \mathrm{Cb}$ \\
\hline 5 & $65 \mathrm{Bb}$ & $76 \mathrm{Ba}$ & $70 \mathrm{Bab}$ & $66 \mathrm{ABb}$ & $94 \mathrm{Aa}$ & $94 \mathrm{Aa}$ & $91 \mathrm{Aab}$ & $85 \mathrm{Ab}$ \\
\hline 6 & $77 \mathrm{ABa}$ & $75 \mathrm{Bab}$ & $75 \mathrm{Bab}$ & $64 \mathrm{ABb}$ & $89 \mathrm{Aa}$ & $92 \mathrm{Aa}$ & $91 \mathrm{Aa}$ & $87 \mathrm{Aa}$ \\
\hline 7 & $81 \mathrm{Aab}$ & $88 \mathrm{Aa}$ & $81 \mathrm{Aab}$ & $72 A b$ & $91 \mathrm{Aa}$ & $95 \mathrm{Aa}$ & $94 \mathrm{Aa}$ & $72 \mathrm{Bb}$ \\
\hline 8 & $77 \mathrm{ABa}$ & $81 \mathrm{ABa}$ & $78 \mathrm{Aa}$ & $61 \mathrm{Bb}$ & $88 \mathrm{Aab}$ & $97 \mathrm{Aa}$ & $87 \mathrm{Aab}$ & $81 \mathrm{ABb}$ \\
\hline 9 & $38 \mathrm{Dab}$ & $52 \mathrm{Ca}$ & $41 \mathrm{Cab}$ & $29 \mathrm{Db}$ & $79 \mathrm{ABa}$ & $83 \mathrm{ABa}$ & $80 \mathrm{ABa}$ & $60 \mathrm{BCb}$ \\
\hline C.V. $(\%$ & \multicolumn{4}{|c|}{9,0} & \multicolumn{4}{|c|}{5,4} \\
\hline
\end{tabular}

$\left({ }^{*}\right)$ Letras maiúsculas: comparações dentro de cada coluna; letras minúsculas: comparações dentro de cada linha, para cada condição de umidade relativa do ar (Teste de Tukey, $5 \%$ ).

TABELA 7 - Cultivar BR-37. Envelhecimento acelerado: dados obtidos para os efeitos de lotes e tamanho das sementes, em testes conduzidos com amostras distribuídas em camada única, durante 72 horas a $41^{\circ} \mathrm{C}$ e diferentes condições de umidade relativa do ar. Piracicaba, 1998/99.

\begin{tabular}{|c|c|c|c|c|c|c|c|c|}
\hline \multirow[t]{2}{*}{ Lotes } & \multicolumn{4}{|c|}{ Sistema Tradicional } & \multicolumn{4}{|c|}{ Solução Salina } \\
\hline & Orig. & 13 & 12 & 10 & Orig. & 13 & 12 & 10 \\
\hline & \multicolumn{8}{|c|}{ - } \\
\hline 1 & $41 \mathrm{Ba}$ & $47 \mathrm{Ca}$ & $52 \mathrm{Ba}$ & $54 \mathrm{Ba}$ & $80 \mathrm{BCa}$ & $74 \mathrm{ABa}$ & $79 \mathrm{ABCa}$ & $75 \mathrm{Aa}$ \\
\hline 2 & $20 \mathrm{CDa}$ & $23 \mathrm{DEa}$ & $27 \mathrm{Ca}$ & $31 \mathrm{Ca}$ & $81 \mathrm{ABCab}$ & $85 \mathrm{ABa}$ & $71 \mathrm{CDb}$ & $75 \mathrm{Aab}$ \\
\hline 3 & $10 \mathrm{Da}$ & $15 \mathrm{Ea}$ & $11 \mathrm{Da}$ & $12 \mathrm{Ea}$ & $37 \mathrm{Db}$ & $58 \mathrm{Ca}$ & $57 \mathrm{Da}$ & $52 \mathrm{Bab}$ \\
\hline 4 & $20 \mathrm{CDa}$ & $17 \mathrm{Eab}$ & $10 \mathrm{Db}$ & $13 \mathrm{Eab}$ & $78 \mathrm{Ca}$ & $77 \mathrm{BCa}$ & $73 \mathrm{BCDa}$ & $50 \mathrm{Bb}$ \\
\hline 5 & $73 \mathrm{Abc}$ & $85 \mathrm{ABa}$ & $83 \mathrm{Aa}$ & $67 \mathrm{Ac}$ & $91 \mathrm{ABCab}$ & $93 \mathrm{Aa}$ & $88 \mathrm{ABab}$ & $79 \mathrm{Ab}$ \\
\hline 6 & $77 \mathrm{Aa}$ & $81 \mathrm{Ba}$ & $83 \mathrm{Aa}$ & $59 \mathrm{ABb}$ & $93 \mathrm{ABa}$ & $93 \mathrm{Aa}$ & $89 \mathrm{ABab}$ & $82 \mathrm{Ab}$ \\
\hline 7 & $77 \mathrm{Aa}$ & $84 \mathrm{Ba}$ & $85 \mathrm{Aa}$ & $60 \mathrm{ABb}$ & $89 \mathrm{ABCa}$ & $91 \mathrm{ABa}$ & $90 \mathrm{Aa}$ & $75 \mathrm{Ab}$ \\
\hline 8 & $75 \mathrm{Ab}$ & $93 \mathrm{Aa}$ & $77 \mathrm{Ab}$ & $63 \mathrm{Ac}$ & $94 \mathrm{Aa}$ & $89 \mathrm{ABab}$ & $89 \mathrm{ABab}$ & $80 \mathrm{Ab}$ \\
\hline 9 & $39 \mathrm{BCa}$ & $41 \mathrm{CDa}$ & $46 \mathrm{Ba}$ & $21 \mathrm{Db}$ & $76 \mathrm{Cab}$ & $77 \mathrm{BCa}$ & $75 \mathrm{BCDab}$ & $66 \mathrm{Abb}$ \\
\hline C.V. $(\%$ & \multicolumn{4}{|c|}{11,3} & \multicolumn{4}{|c|}{7,6} \\
\hline
\end{tabular}

${ }^{*}$ ) Letras maiúsculas: comparações dentro de cada coluna; letras minúsculas: comparações dentro de cada linha, para cada condição de umidade relativa do ar (Teste de Tukey, $5 \%$ ).

Scientia Agricola, v.57, n.3, p.473-482, jul./set. 2000 
diferenças mais definidas entre os potenciais fisiológicos dos lotes, sem a alternância de comportamento geralmente observada em lotes de qualidade intermediária. Por outro lado, os graus de umidade das sementes envelhecidas durante 72 horas, sob a influência de solução salina variaram de $12,2 \%$ a $12,5 \%$; assim, nota-se que o ponto de equilíbrio das sementes com a umidade relativa do ar já havia sido alcançado durante as 48 horas de envelhecimento.

Os efeitos do tamanho se manifestaram de acordo com a tendência relatada anteriormente, ou seja, as sementes menores (10x3/4") exibiram desempenho significativamente inferior em sete (distribuição após pesagem) ou seis (camada única) dos nove lotes avaliados. Esta observação também poderia ser explicada pelo fato dessas sementes captarem maior quantidade de água durante os períodos de envelhecimento. No entanto, nas condições específicas do teste conduzido durante 72 horas, a diferença entre os graus de umidade foram muito pouco expressivas, variando de $28,4 \%$ a $29,2 \%$, considerando-se os dois procedimentos adotados para a distribuição das amostras. Isto conduziu a duas observações adicionais:

a) as diferenças detectadas no potencial fisiológico correspondem à deficiência no desempenho das sementes retidas na peneira $10 \times 3 / 4$ ", em relação às de tamanho médio, e não ao possível efeito do tamanho da semente sobre sua resposta às condições de temperatura e umidade relativa do ar, durante o teste de envelhecimento acelerado;

b) é provável que o uso de amostras com massa uniforme realmente promova a redução de diferenças na velocidade e, principalmente, na intensidade de captação de água durante o teste, desde que seja adotado o período de 72 horas. Caso esta hipótese esteja correta, os resultados são condizentes com as observações efetuadas por Tomes et al. (1988), pois nos EUA é recomendada a condução do teste de envelhecimento acelerado durante 72 horas; no Brasil, normalmente se utiliza o período de 48 horas (Marcos Filho et al., 1987).

\section{Testes conduzidos com sementes de 'Embrapa 48'}

Germinação - Houve separação dos lotes de acordo com diferenças no potencial fisiológico, com destaque para os lotes $4 \mathrm{~A}$ e $5 \mathrm{~A}$, tanto no material original como nos classificados pelo tamanho. Os lotes $1 \mathrm{~A}, 2 \mathrm{~A}$ e $3 \mathrm{~A}$ apresentaram germinação inferior à dos demais, situação que se evidenciou nas sementes classificadas (TABELA 8), enquanto os lotes $6 \mathrm{~A}$ e $7 \mathrm{~A}$ mostraram comportamento próximo dos de melhor qualidade.

Assim, da mesma forma relatada para o cultivar BR-37, foi atingido o objetivo proposto inicialmente, ou seja, a utilização de lotes que diferissem quanto ao potencial fisiológico, mas compatíveis com os padrões estabelecidos para a comercialização de sementes de soja. A observação dos dados referentes ao material
TABELA 8 - Cultivar Embrapa 48. Germinação: dados obtidos para os efeitos de lotes, de tamanhos das sementes e coeficiente de variação. Piracicaba, 1998/99.

\begin{tabular}{|c|c|c|c|c|}
\hline \multirow[t]{2}{*}{ Lotes } & \multicolumn{4}{|c|}{ Tamanhos } \\
\hline & Original & 14 & 13 & 11 \\
\hline & \multicolumn{4}{|c|}{-- } \\
\hline $1 \mathrm{~A}$ & $73 \mathrm{Cb}$ & $84 \mathrm{Ba}$ & 77 DEab & $73 \mathrm{CDb}$ \\
\hline $2 \mathrm{~A}$ & $83 \mathrm{BCa}$ & $84 \mathrm{Ba}$ & $68 \mathrm{~Eb}$ & $66 \mathrm{Db}$ \\
\hline $3 A$ & $84 \mathrm{BCa}$ & $83 \mathrm{Ba}$ & $86 \mathrm{CDa}$ & $81 \mathrm{BCa}$ \\
\hline $4 \mathrm{~A}$ & $95 \mathrm{Aa}$ & $96 \mathrm{Aa}$ & $95 \mathrm{ABa}$ & $95 \mathrm{Aa}$ \\
\hline $5 \mathrm{~A}$ & $96 \mathrm{Aa}$ & $96 \mathrm{Aa}$ & $97 \mathrm{Aa}$ & $91 \mathrm{ABa}$ \\
\hline $6 \mathrm{~A}$ & $89 \mathrm{ABab}$ & $95 \mathrm{Aa}$ & $87 \mathrm{BCDab}$ & $86 \mathrm{ABb}$ \\
\hline $7 \mathrm{~A}$ & $90 \mathrm{ABa}$ & $91 \mathrm{ABa}$ & $93 \mathrm{ABCa}$ & $91 \mathrm{ABa}$ \\
\hline
\end{tabular}

$\left.{ }^{*}\right)$ Letras maiúsculas: comparações dentro de cada coluna; letras minúsculas: comparações dentro de cada linha (Teste de Tukey, $5 \%$ de probabilidade).

original permite constatar que apenas o lote $1 \mathrm{~A}$ não atingiu o padrão mínimo desejado. Sabe-se que a avaliação do vigor de sementes deve ser dirigida, preferencialmente, às amostras com germinação semelhante e/ou superior ao padrão mínimo estabelecido para a comercialização (Powell, 1986; Marcos Filho, 1999).

Os efeitos do tamanho das sementes foram menos pronunciados que os constatados para 'BR-37', pois as sementes retidas na peneira $11 \times 3 / 4$ " apresentaram germinação significativamente inferior às da peneira $14 \times 3 / 4$ " em apenas três dos sete lotes avaliados, isto é, em $43 \%$ dos lotes.

Envelhecimento acelerado (48 horas) - a detecção de diferenças no potencial fisiológico das sementes de 'EMBRAPA 48' foi mais evidente no teste de envelhecimento acelerado (48 horas) que no de germinação, embora as principais tendências tenham sido mantidas. O exame da TABELA 9 permite verificar que os lotes foram separados em dois grupos, de acordo com seu desempenho: 0 de qualidade mais alta (lotes 4A, 5A, 6A e 7A) e 0 de desempenho inferior (lotes $1 A, 2 A$ e $3 A$ ). Desta forma, praticamente não foi detectado 0 comportamento característico de lotes com potencial fisiológico intermediário. Quanto aos efeitos do tamanho, as sementes retidas na peneira $11 \times 3 / 4$ " apresentaram menor vigor em dois dos sete lotes avaliados.

De um modo geral, as sementes de 'Embrapa 48' apresentaram potencial fisiológico superior, em relação às de 'BR-37'; provavelmente, as causas dessa diferença estejam relacionadas às hipóteses levantadas durante os comentários referentes à análise da distribuição do tamanho das sementes. Ao mesmo tempo, em lotes de qualidade mais elevada, os efeitos do tamanho das sementes sobre a germinação e o vigor se tornaram menos intensos ou não se manifestaram. Esse fato pode 
ser confirmado ao ser verificado que as sementes da peneira $11 \times 3 / 4$ " apresentaram desempenho comparativamente diferente ao das sementes maiores, nos lotes $1 \mathrm{~A}$ e $3 \mathrm{~A}$, considerados de menor qualidade.

Por outro lado, a diferença no grau de umidade das sementes retidas nas peneiras 14×3/4" e $11 \times 3 / 4$ " foi, em geral, de $0,7 \%$, de modo que esse parâmetro não permite explicar as variações observadas nas respostas ao teste de envelhecimento acelerado, na presente pesquisa.

Verificou-se, novamente, que a utilização de solução salina tornou menos drásticos os efeitos das condições do teste de envelhecimento acelerado, confirmando as considerações efetuadas para 'BR-37'. Paralelamente, não foi constatada redução da eficiência do teste para a identificação de diferenças no potencial fisiológico dos lotes avaliados (TABELAS 9 e 10). Neste caso, porém, observou-se que o desempenho das sementes da peneira $11 \times 3 / 4$ " foi inferior às da peneira $14 \times 3 / 4$ ", para os lotes $1 \mathrm{~A}$ e $2 \mathrm{~A}$.

Envelhecimento acelerado (72 horas) - Os resultados obtidos nesse teste confirmaram a maior qualidade das sementes de 'Embrapa 48' em relação às de 'BR-37', pois o período de 72 horas provocou efeitos menos drásticos sobre a germinação das sementes envelhecidas.

As TABELAS 11 e 12 mostram que a separação dos lotes quanto ao potencial fisiológico não diferiu da proporcionada pelo período de 48 horas. O mesmo ocorreu quando foi utilizada solução salina. Foram, também, detectados efeitos do tamanho das sementes sobre o seu desempenho, com desvantagem para as retidas na peneira $11 \times 3 / 4 "$, coincidentemente em lotes com desempenho inferior. Essa ocorrência também não pode ser justificada por diferenças no grau de umidade das sementes, quando as amostras foram distribuídas após pesagem.

TABELA 9 - Cultivar Embrapa 48. Envelhecimento acelerado: dados obtidos para os efeitos de lotes e tamanho das sementes, em testes conduzidos com amostras pré-pesadas, durante 48 horas a $41^{\circ} \mathrm{C}$ e diferentes condições de umidade relativa do ar. Piracicaba, 1998/99.

\begin{tabular}{|c|c|c|c|c|c|c|c|c|}
\hline \multirow[t]{2}{*}{ Lotes } & \multicolumn{4}{|c|}{ Sistema Tradicional } & \multicolumn{4}{|c|}{ Solução Salina } \\
\hline & Orig. & 14 & 13 & 11 & Orig. & 14 & 13 & 11 \\
\hline & \multicolumn{8}{|c|}{ - } \\
\hline $1 \mathrm{~A}$ & $60 \mathrm{Cb}$ & $73 \mathrm{Ba}$ & $66 \mathrm{Cab}$ & $46 \mathrm{Cc}$ & $83 \mathrm{BCDa}$ & $85 \mathrm{BCa}$ & $85 \mathrm{Ca}$ & $74 \mathrm{Bb}$ \\
\hline $2 \mathrm{~A}$ & $69 \mathrm{BCa}$ & $73 \mathrm{Ba}$ & $75 \mathrm{Ba}$ & $68 \mathrm{Ba}$ & $78 \mathrm{Dab}$ & $77 \mathrm{Cab}$ & $84 \mathrm{Ba}$ & $76 \mathrm{Bb}$ \\
\hline $3 A$ & $72 \mathrm{Bab}$ & $79 \mathrm{Ba}$ & $77 \mathrm{Ba}$ & $68 \mathrm{Bb}$ & $87 \mathrm{BCa}$ & $87 \mathrm{Ba}$ & $87 \mathrm{Ba}$ & $83 \mathrm{Ba}$ \\
\hline $4 \mathrm{~A}$ & $95 \mathrm{Aa}$ & $96 \mathrm{Aa}$ & $97 \mathrm{Aa}$ & $95 \mathrm{Aa}$ & $96 \mathrm{ABa}$ & $99 \mathrm{Aa}$ & $97 \mathrm{Aa}$ & $97 \mathrm{Aa}$ \\
\hline $5 A$ & $91 \mathrm{Aa}$ & $94 \mathrm{Aa}$ & $95 \mathrm{Aa}$ & $90 \mathrm{Aa}$ & $97 \mathrm{Aa}$ & $97 \mathrm{Aa}$ & $98 \mathrm{Aa}$ & $93 \mathrm{Aa}$ \\
\hline $6 \mathrm{~A}$ & $96 \mathrm{Aa}$ & $95 \mathrm{Aa}$ & $94 \mathrm{Aa}$ & $94 \mathrm{Aa}$ & $94 \mathrm{ABa}$ & $96 \mathrm{ABa}$ & $99 \mathrm{Aa}$ & $97 \mathrm{Aa}$ \\
\hline $7 \mathrm{~A}$ & $94 \mathrm{Aa}$ & $92 \mathrm{Aa}$ & $95 \mathrm{Aa}$ & $92 \mathrm{Aa}$ & $98 \mathrm{Aa}$ & $96 \mathrm{ABa}$ & $99 \mathrm{Aa}$ & $95 \mathrm{Aa}$ \\
\hline C.V. $(\%$ & \multicolumn{4}{|c|}{6,3} & \multicolumn{4}{|c|}{6,7} \\
\hline
\end{tabular}

$\left({ }^{\star}\right)$ Letras maiúsculas: comparações dentro de cada coluna; letras minúsculas: comparações dentro de cada linha, para cada condição de umidade relativa do ar (Teste de Tukey, $5 \%$ ).

TABELA 10 - Cultivar Embrapa 48. Envelhecimento acelerado: dados obtidos para os efeitos de lotes e tamanho das sementes, em testes conduzidos com amostras distribuídas em camada única, durante 48 horas a $41^{\circ} \mathrm{C}$ e diferentes condições de umidade relativa do ar. Piracicaba, 1998/99.

\begin{tabular}{|c|c|c|c|c|c|c|c|c|}
\hline \multirow[t]{2}{*}{ Lotes } & \multicolumn{4}{|c|}{ Sistema Tradicional } & \multicolumn{4}{|c|}{ Solução Salina } \\
\hline & Orig. & 14 & 13 & 11 & Orig. & 14 & 13 & 11 \\
\hline & & 6 & 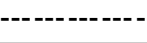 & - & & $\ldots$ & $\cdots$ & ------ \\
\hline $1 \mathrm{~A}$ & $62 \mathrm{Db}$ & $71 \mathrm{Da}$ & $73 \mathrm{Ba}$ & $70 \mathrm{Bab}$ & $66 \mathrm{Cb}$ & $81 \mathrm{Ca}$ & $67 \mathrm{Bb}$ & $64 \mathrm{Cb}$ \\
\hline $2 \mathrm{~A}$ & $79 \mathrm{BCa}$ & $82 \mathrm{BCa}$ & $81 \mathrm{Ba}$ & $74 \mathrm{Ba}$ & $79 \mathrm{Bab}$ & $83 \mathrm{BCa}$ & $76 \mathrm{Bab}$ & $69 \mathrm{Cb}$ \\
\hline $3 A$ & $70 \mathrm{CDa}$ & $73 \mathrm{CDa}$ & $75 \mathrm{Ba}$ & $76 \mathrm{Ba}$ & $79 \mathrm{Ba}$ & $81 \mathrm{Ca}$ & $75 \mathrm{Ba}$ & $81 \mathrm{Ba}$ \\
\hline $4 \mathrm{~A}$ & $94 \mathrm{Aa}$ & $93 \mathrm{ABa}$ & $95 \mathrm{Aa}$ & $94 \mathrm{Aa}$ & $97 \mathrm{Aa}$ & $97 \mathrm{Aa}$ & $96 \mathrm{Aa}$ & $94 \mathrm{Aa}$ \\
\hline $5 \mathrm{~A}$ & $92 \mathrm{Aa}$ & $89 \mathrm{ABa}$ & $94 \mathrm{Aa}$ & $95 \mathrm{Aa}$ & $97 \mathrm{Aa}$ & $97 \mathrm{Aa}$ & $96 \mathrm{Aa}$ & $94 \mathrm{Aa}$ \\
\hline $6 \mathrm{~A}$ & $91 \mathrm{Aa}$ & $95 \mathrm{Aa}$ & $95 \mathrm{Aa}$ & $95 \mathrm{Aa}$ & $96 \mathrm{Aa}$ & $94 \mathrm{Aa}$ & $97 \mathrm{Aa}$ & $94 \mathrm{Aa}$ \\
\hline $7 \mathrm{~A}$ & $93 \mathrm{Aa}$ & $95 \mathrm{Aa}$ & $95 \mathrm{Aa}$ & $94 \mathrm{Aa}$ & $94 \mathrm{Aa}$ & $93 \mathrm{Aa}$ & $93 \mathrm{Aa}$ & $96 \mathrm{Aa}$ \\
\hline
\end{tabular}

$\left(^{*}\right)$ Letras maiúsculas: comparações dentro de cada coluna; letras minúsculas: comparações dentro de cada linha, para cada condição de umidade relativa do ar (Teste de Tukey, $5 \%$ ). 
TABELA 11 - Cultivar Embrapa 48. Envelhecimento acelerado: dados obtidos para os efeitos de lotes e tamanho das sementes, em testes conduzidos com amostras pré-pesadas, durante 72 horas a $41^{\circ} \mathrm{C}$ e diferentes condições de umidade relativa do ar. Piracicaba, 1998/99.

\begin{tabular}{|c|c|c|c|c|c|c|c|c|}
\hline \multirow[t]{2}{*}{ Lotes } & \multicolumn{4}{|c|}{ Sistema Tradicional } & \multicolumn{4}{|c|}{ Solução Salina } \\
\hline & Orig. & 14 & 13 & 11 & Orig. & 14 & 13 & 11 \\
\hline & \multicolumn{8}{|c|}{ } \\
\hline $1 \mathrm{~A}$ & $25 \mathrm{Db}$ & $31 \mathrm{Dab}$ & $37 \mathrm{BCa}$ & $35 \mathrm{Ca}$ & $76 \mathrm{Bab}$ & $83 \mathrm{Ba}$ & $73 \mathrm{Cb}$ & $65 \mathrm{Cc}$ \\
\hline $2 \mathrm{~A}$ & $45 \mathrm{BCa}$ & $41 \mathrm{BCa}$ & $38 \mathrm{Ba}$ & $22 \mathrm{Db}$ & $77 \mathrm{Ba}$ & $79 \mathrm{Ba}$ & $77 \mathrm{BCa}$ & $75 \mathrm{Ba}$ \\
\hline $3 A$ & $37 \mathrm{Ca}$ & $32 \mathrm{Ca}$ & $28 \mathrm{Ca}$ & $19 \mathrm{Db}$ & $85 \mathrm{Ba}$ & $87 \mathrm{Ba}$ & $86 \mathrm{Ba}$ & $80 \mathrm{Ba}$ \\
\hline $4 \mathrm{~A}$ & $85 \mathrm{Aa}$ & $93 \mathrm{Aa}$ & $88 \mathrm{Aa}$ & $87 \mathrm{Aa}$ & $97 \mathrm{Aa}$ & $99 \mathrm{Aa}$ & $96 \mathrm{Aa}$ & $95 \mathrm{Aa}$ \\
\hline $5 A$ & $85 \mathrm{Aa}$ & $91 \mathrm{Aa}$ & $89 \mathrm{Aa}$ & $89 \mathrm{Aa}$ & $95 \mathrm{Aa}$ & $98 \mathrm{Aa}$ & $98 \mathrm{Aa}$ & $95 \mathrm{Aa}$ \\
\hline $6 \mathrm{~A}$ & $89 \mathrm{Aa}$ & $88 \mathrm{Aa}$ & $89 \mathrm{Aa}$ & $89 \mathrm{Aa}$ & $97 \mathrm{Aa}$ & $95 \mathrm{ABa}$ & $95 \mathrm{ABa}$ & $93 \mathrm{Aa}$ \\
\hline $7 \mathrm{~A}$ & $82 \mathrm{Aab}$ & $91 \mathrm{Aa}$ & $89 \mathrm{Aa}$ & $71 \mathrm{Bb}$ & $96 \mathrm{Aa}$ & $96 \mathrm{ABa}$ & $97 \mathrm{Aa}$ & $94 \mathrm{Aa}$ \\
\hline C.V. $(\%$ & \multicolumn{4}{|c|}{7,0} & \multicolumn{4}{|c|}{6,5} \\
\hline
\end{tabular}

$\left(^{*}\right)$ Letras maiúsculas: comparações dentro de cada coluna; letras minúsculas: comparações dentro de cada linha, para cada condição de umidade relativa do ar (Teste de Tukey, $5 \%$ ).

TABELA 12 - Cultivar Embrapa 48. Envelhecimento acelerado: dados obtidos para os efeitos de lotes e tamanho das sementes, em testes conduzidos com amostras distribuídas em camada única, durante 72 horas a $41^{\circ} \mathrm{C}$ e diferentes condições de umidade relativa do ar. Piracicaba, 1998/99.

\begin{tabular}{|c|c|c|c|c|c|c|c|c|}
\hline \multirow[t]{2}{*}{ Lotes } & \multicolumn{4}{|c|}{ Sistema Tradicional } & \multicolumn{4}{|c|}{ Solução Salina } \\
\hline & Orig. & 14 & 13 & 11 & Orig. & 14 & 13 & 11 \\
\hline & \multicolumn{8}{|c|}{ - } \\
\hline $1 \mathrm{~A}$ & $47 \mathrm{Cab}$ & $49 \mathrm{Ca}$ & $38 \mathrm{Db}$ & $37 \mathrm{Db}$ & $77 \mathrm{Ba}$ & $77 \mathrm{Ba}$ & $73 \mathrm{Ba}$ & $75 \mathrm{BCa}$ \\
\hline $2 \mathrm{~A}$ & $62 \mathrm{Ba}$ & $63 \mathrm{Ba}$ & $58 \mathrm{Bab}$ & $51 \mathrm{Cb}$ & $79 \mathrm{Ba}$ & $78 \mathrm{Bab}$ & $73 \mathrm{Bab}$ & $69 \mathrm{Cb}$ \\
\hline $3 A$ & $45 \mathrm{Ca}$ & $47 \mathrm{Ca}$ & $47 \mathrm{Ca}$ & $43 \mathrm{CDa}$ & $85 \mathrm{ABab}$ & $80 \mathrm{Bab}$ & $91 \mathrm{Aa}$ & $81 \mathrm{Bb}$ \\
\hline $4 \mathrm{~A}$ & $89 \mathrm{Aa}$ & $87 \mathrm{Aa}$ & $91 \mathrm{Aa}$ & $86 \mathrm{Ba}$ & $95 \mathrm{Aa}$ & $97 \mathrm{Aa}$ & $98 \mathrm{Aa}$ & $95 \mathrm{Aa}$ \\
\hline $5 A$ & $89 \mathrm{Aa}$ & $85 \mathrm{Aa}$ & $90 \mathrm{Aa}$ & $87 \mathrm{Aa}$ & $95 \mathrm{Aa}$ & $97 \mathrm{Aa}$ & $92 \mathrm{Aa}$ & $95 \mathrm{Aa}$ \\
\hline $6 \mathrm{~A}$ & $90 \mathrm{Aa}$ & $83 \mathrm{Aa}$ & $92 \mathrm{Aa}$ & $85 \mathrm{Aa}$ & $96 \mathrm{Aa}$ & $99 \mathrm{Aa}$ & $96 \mathrm{Aa}$ & $98 \mathrm{Aa}$ \\
\hline $7 \mathrm{~A}$ & $91 \mathrm{Aa}$ & $85 \mathrm{Aa}$ & $91 \mathrm{Aa}$ & $83 \mathrm{Ba}$ & $93 \mathrm{Aa}$ & $99 \mathrm{Aa}$ & $99 \mathrm{Aa}$ & $94 \mathrm{Aa}$ \\
\hline C.V.(\%) & \multicolumn{4}{|c|}{7,0} & \multicolumn{4}{|c|}{6,4} \\
\hline
\end{tabular}

$\left(^{*}\right)$ Letras maiúsculas: comparações dentro de cada coluna; letras minúsculas: comparações dentro de cada linha, para cada condição de umidade relativa do ar (Teste de Tukey, $5 \%$ ).

\section{Comentário Geral}

A descrição da metodologia utilizada para a condução do teste de envelhecimento acelerado relata dois procedimentos para a distribuição das amostras na superfície da tela metálica, ou seja, após pré-pesagem ou formando camada simples. Deve ser destacado que, embora o grau de umidade das sementes menores tenha superado de maneira mais acentuada o das maiores, quando as amostras foram distribuídas para constituir uma camada simples na superfície da tela metálica, as informações proporcionadas foram tão consistentes quanto as obtidas com a utilização de amostras com massa uniforme.

Essa observação se refere aos efeitos do tamanho das sementes e à detecção de diferenças quanto ao potencial fisiológico dos lotes de 'BR-37' e 'Embrapa 48'. Assim, as diferenças no grau de umidade não se traduziram no desempenho das sementes no teste de envelhecimento acelerado.
É possível que essas diferença entre os graus de umidade de sementes com tamanhos distintos, observadas ao longo da presente pesquisa, não sejam suficientes para provocar variações no desempenho das sementes submetidas ao envelhecimento acelerado. Conforme comentário anterior, Fahim \& McDonald ${ }^{1}$ consideraram que o grau de umidade das sementes com diferentes tamanhos deve ser determinado ao final do período de envelhecimento porque o efeito desse parâmetro poderia se traduzir nos resultados. No entanto, esses autores trabalharam com variações mais acentuadas no tamanho de sementes de soja que as verificadas na presente pesquisa, ou seja, massa de 215,4 g/1000 sementes "grandes" e 108,7 g/ 1000 sementes "pequenas", apresentando, ao final de 72 horas de envelhecimento, $28,8 \%$ e $22,9 \%$ de água, respectivamente. A diferença entre as massas de 1000 sementes entre as sementes maiores $(14 \times 3 / 4 ")$ e as menores $\left(10 \times 3 / 4^{\prime}\right)$, determinada no presente trabalho, variou de 150 a $170 \mathrm{~g} / 1000$ sementes, para ambos os cultivares. 
Os resultados referentes ao segundo ano experimental, também não apresentados, confirmaram os obtidos no primeiro. Destaque-se que, de um modo geral, os lotes utilizados no segundo ano apresentaram potencial fisiológico superior ao determinado no ano anterior, para ambos os cultivares, provavelmente devido às condições climáticas mais favoráveis durante o desenvolvimento das plantas e formação das sementes; essa hipótese pode ser confirmada pelo fato de que, no segundo ano, a massa de 1000 sementes dos dois cultivares foi semelhante, tanto quando se compararam os materiais originais como as diferentes classes de tamanho, após a separação das sementes em peneiras de crivos oblongos.

Consequentemente, as observações efetuadas permitiram que as conclusões da presente pesquisa fossem baseadas nos resultados dos dois anos experimentais.

\section{CONCLUSÕES}

- A distribuição de amostras com massa uniforme ou constituindo camada única na superfície da tela metálica colocada no interior da caixa plástica, utilizada como compartimento individual no teste de envelhecimento acelerado, pode atenuar mas não elimina os efeitos do tamanho sobre o comportamento das sementes no teste.

- O teste de envelhecimento acelerado, em sementes de soja, fornece informações mais consistentes quando as amostras comparadas são constituídas por sementes de tamanho uniforme.

- O uso de solução salina no interior do compartimento individual, promovendo a redução da umidade relativa, torna o teste de envelhecimento acelerado menos severo, mas não reduz sua eficiência em relação ao procedimento tradicional.

\section{REFERÊNCIAS BIBLIOGRÁFICAS}

BRASIL. Ministério da Agricultura. Regras para análise de sementes. Brasília: Departamento Nacional de Produção Vegetal, 1992. 365p.
DELOUCHE, J.C. An accelerated aging technique for predicting the relative storability of crimson clover and tall fescue seed lots. Agronomy Abstracts 1965, p.40, 1965.

EGLI, D.B. Seed water relations and the regulation of the duration of seed growth in soybean. Journal of Experimental Botany, v.41, p.243-248, 1990.

EMBRAPA. Centro Nacional de Pesquisa de Soja. Recomendações para a cultura da soja no Paraná 1996/ 97. Londrina: Embrapa-Soja, 1996. 187p. (Documentos, 97).

JIANHUA, Z.; McDONALD, M.B. The saturated salt accelerated aging test for small seeds. Seed Science and Technology, v.25, p.123-131, 1996.

MARCOS FILHO, J. Testes de vigor: importância e utilização. In: KRZYZANOWSKI, F.C.; VIEIRA, R.D.; FRANÇA NETO, J.B. Vigor de sementes: conceitos e testes. Londrina: Associação Brasileira de Tecnologia de Sementes, Comitê de Vigor de Sementes, 1999. cap. 1, p.1-21.

MARCOS FILHO, J.; CICERO, S.M.; SILVA, W.R. Avaliação da qualidade de sementes. Piracicaba: FEALQ, 1987. 230p.

KRZYZANOWSKI, F.C.; FRANÇA NETO, J.B.; COSTA, N.P. Efeito da classificação de sementes de soja pelo tamanho sobre sua qualidade e precisão da semeadura. Revista Brasileira de Sementes, v.25, p.59-68, 1991.

PANOBIANCO, M.; MARCOS FILHO, J. Comparação entre métodos para avaliação da qualidade fisiológica de sementes de pimentão. Revista Brasileira de Sementes, v.20, p.306-310, 1998.

POWELL, A.A. Cell membranes and seed leachate conductivity in relation to the quality of seed for sowing. Journal of Seed Technology, v.10, p.81-100, 1986.

TeKRONY, D.M. Accelerated aging test. In: HAMPTON, J.G. e TeKRONY, D.M. (Ed.) Handbook of vigour test methods. 3. ed. Zurich: International Seed Testing Association, 1995. p.35-50.

TOMES, L.J.; TeKRONY, D.M.; EGLI, D.B. Factors influencing the tray accelerated aging test for soybean seed. Journal of Seed Technology, v.12, p.24-35, 1988.

VIEIRA, R.D.; TeKRONY, D.M.; EGLI, D.B. Effect of drought and defoliation stress in the field on soybean seed germination and vigor. Crop Science, v.32, p.471-475, 1992.

WETZEL, C.T. Efeito do tamanho das sementes de soja. In: SEMINÁRIO NACIONAL DE PESQUISA DE SOJA,1., Londrina, 1978. Anais. Londrina: EMBRAPA, CNPSo, 1978. p.333-341.

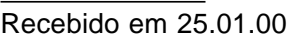

